\title{
Pain in patients attending outpatient rehabilitation: results of a pilot study
}

\author{
Francesca Cecchi • Anita Paperini · Raffaello Molino Lova • \\ Guido Pasquini · Roberta Boni - Chiara Castagnoli • \\ Federica Vannetti - Luca Padua · Claudio Macchi
}

Received: 31 October 2014 / Accepted: 16 January 2015/Published online: 10 February 2015

(c) SIMI 2015

\begin{abstract}
The aim of the study was to investigate pain occurrence, characteristics and correlations in an outpatient rehabilitation setting. This was an observational pilot study. The setting was an outpatient rehabilitation facility. The subjects included all patients attending physiotherapy in the week 25th to 29th September 2010 and the interventions were made using self-administered questionnaire. Ongoing pain was assessed by a yes-no question, pain intensity by a numeric rating scale (NRS) ranging $0-10$. Pain-related medication was investigated, along with pain characteristics, patient treatment expectations, life satisfaction, and catastrophism. Of the 201 patients, 12 were excluded and 189 enrolled (age 63.6 $\pm 15.6 ; 70.4 \%$ women). Pain (mean NRS $=5.6 \pm 2.4$ ) was reported by $60.9 \%$ patients (66\% orthopedic and $40 \%$ neurological). In $87.8 \%$ cases, pain was chronic ( $>6$ months). According to patients reporting pain, the main objectives of treatment
\end{abstract}

F. Cecchi · A. Paperini $(\bowtie) \cdot$ R. Molino Lova $\cdot$ G. Pasquini ·

R. Boni - C. Castagnoli - F. Vannetti - L. Padua - C. Macchi

Fondazione Don Carlo Gnocchi, Scientific Institute, Florence,

Italy

e-mail: anitapaperini@hotmail.it

F. Cecchi

e-mail: francescacecchi2002@libero.it

R. Molino Lova

e-mail: raffmoli@tin.it

G. Pasquini

e-mail: pasquinifam@hotmail.com

R. Boni

e-mail: boni.roberta@virgilio.it

C. Castagnoli

e-mail: chiara.castagnoli@virgilio.it

F. Vannetti

e-mail: federica.vannetti@unifi.it were both pain relief and functional recovery for $51 \%$; pain relief for $24.9 \%$; functional recovery for $22.8 \%$. Low treatment expectations were reported by $15.3 \%$ patients; catastrophism by $40.7 \% ; 28.6 \%$ patients were on pain medication: use of drugs was related to age $(p=0.005)$, pain intensity ( $p=0.009)$ and catastrophism $(p=0.0003)$. In a multivariate analysis, pain was independently correlated with an orthopedic versus neurological diagnosis $(p=0.000)$, and with reduced treatment expectations $(p=0.020)$, while independent of age $(p=0.74)$ gender $(p=0.22)$, and catastrophism (0.17). A high prevalence of pain was observed in outpatients undergoing rehabilitation. Pain was chronic in most cases. Pain relief was the most desired treatment outcome by patients reporting pain. Pain complaint was independently correlated to orthopedic vs neurological diagnosis and to reduced treatment expectations.

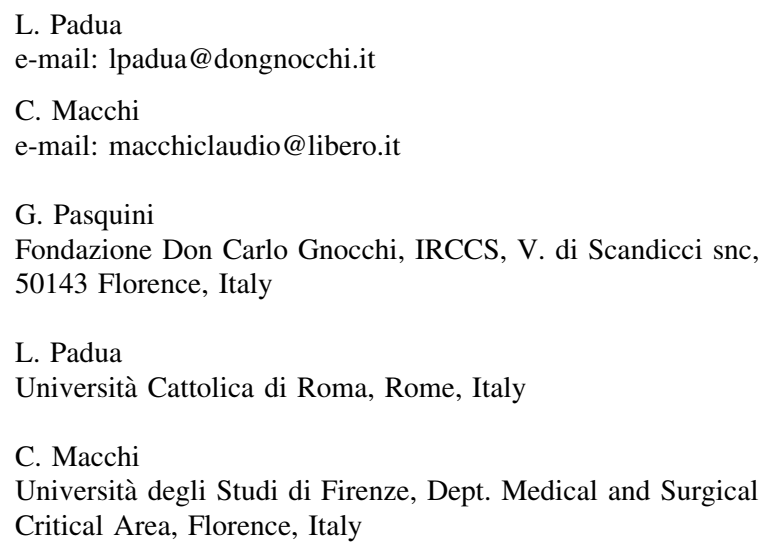


Keywords Pain - Rehabilitation - Pain assessment - Pain management $\cdot$ Pain medication $\cdot$ Chronic pain

\section{Introduction}

Pain can cause the impairment of physical health and function, and is a common symptom in patients undergoing rehabilitation [1]. Pain is defined by the International Association for the Study of Pain (IASP) as: "an unpleasant sensory and emotional experience, which we primarily associate with tissue damage or describe in terms of such damage, or both;" [2] pain that restricts function may be the main indication for rehabilitation or may often complicate a disabling disease [3].

Pain is reported in many chronic illnesses, and pain may hinder functional recovery. Recent studies suggest that pain may predict a poor rehabilitation outcome [4]. From the patient's perspective, pain relief is often the most important element of satisfaction after rehabilitation. According to the International Classification of Functioning, Disability and Health (ICF), each individual, depending on the medical condition, physical limitations, personality and style of interaction with the opportunities offered by the environment, develops a personal assessment of what a good quality of life may be, and consequently what are the objectives of a program of rehabilitation [5]. Therefore, early detection and monitoring of a pain complaint in any clinical setting including rehabilitation is universally recognized as a relevant clinical issue [6,7]. This is also expressed by recent legislation on pain in Italy and Portugal and by the development of European-wide policy strategies and activities for improved pain care in Europe [8].

A relevant issue for correct pain assessment and management is the verification of whether reported pain is acute, subacute or chronic. Chronic pain has been described by the International Society of interventional Pain Physicians (ASIPP) as: "pain that persists 6 months after the injury and beyond the usual course of an acute disease" [9]. While acute and subacute pain are often related to strictly organic factors, and better treated with drugs and physical therapy, chronic pain is very difficult to treat [9]. Indeed, chronic pain patients become more resistant to therapy, and their use of health services is up to five times higher than the use by the general population [10]. Further, chronic pain ranks among the top three disabling conditions in developed countries [11]. According to the biopsycho-social model, describing chronic pain as the result of a complex interaction of psychological, social and organic determinants, chronic pain management should involve a multidimensional assessment and a multidisciplinary approach [2]. International guidelines recommend that the biological, psychological and social factors must all be addressed simultaneously, but this multidisciplinary simultaneous approach is not widely diffused in clinical and rehabilitation practice.

The Don Gnocchi Foundation is a no-profit rehabilitation institution including 29 Centers in several Italian Regions. The optimal assessment and treatment of the patient in pain is a highly debated issue, thus in Florence and Rome we developed the project of investigating pain reports, characteristics and management in patients attending different rehabilitation settings. A number of centers were involved throughout Italy as part of a continuous quality improvement process. The pilot study of this multicentric research project describes the study protocol and preliminary results collected, investigating pain reports, characteristics and correlations in patients attending rehabilitation in a large outpatient rehabilitation department in Florence. Possible pain correlations included demographics, clinical diagnosis, use of drugs, and some psychological characteristics, including catastrophism and treatment expectations.

\section{Methods}

\section{Study sample}

All patients in outpatient rehabilitation in the week 25th to 29th, September 2010 at the Don Gnocchi Foundation Outpatient rehabilitation department of Florence were asked to fill in a self-administered questionnaire. Exclusion criteria were cognitive impairment (Mini Mental State Examination <24/31) [12], and aphasia.

\section{Measures}

A self-administered questionnaire was presented to all the patients who consented to be interviewed. The questionnaire included basic socio-demographic information and the first question of the Brief Pain Inventory (BPI), Italian version [13]: for those who answered 'yes' (Do you feel any pain at this moment?), two further questions taken from the BPI were put to them: concerning specifically current pain intensity, measured by a numeric rating scale (NRS, range 0-100) [14, 15] and pain-related use of drugs. If the patient reported pain-related use of drugs, we investigated the drug type and prescription. The main goal of undergoing treatment, according to the patient, was investigated with an open question ("what is your main objective in undergoing this rehabilitation treatment?") Other questions concerned expectations regarding the treatment; life satisfaction [16] and catastrophism, scored with a question taken from the Pain Catastrophizing Scale [17, 18]. 
Statistical analysis

Statistical analysis was performed using the STATA 7.0 software (Stata Corporation, College Station, Texas, USA). Data are presented as the mean $\pm \mathrm{SD}$ or as the absolute number, followed by the percentage in brackets. Baseline differences across groups were compared using the ANOVA, the Chi-square test or the Kruskal-Wallis rank test for continuous, categorical or ordinal variables, respectively. A multivariable logistic analysis was carried out to assess independent correlations of ongoing pain in the study population. Estimates of association are presented as odd ratios (ORs), along with $95 \%$ confidence intervals. Type 1 error was set at the two-sided 0.05 level.

\section{Results}

Of the 201 patients, 12 were excluded because of severe cognitive impairment (7) or aphasia. (4) The 189 enrolled patients had a mean age 63.6 years (SD 17.7), $70.4 \%$ women. Patients' characteristics are summarized in Table 1.

A minority of patients $(21.2 \%)$ were being treated for a neurological disorder such as hemiplegia/hemiparesis

Table 1 Patients' characteristics

\begin{tabular}{ll}
\hline & Patients $(n=189)$ \\
\hline Age (Years, M + SD) & $63.6 \pm 17.7$ \\
Male $(n)$ & 56 \\
Female $(n)$ & 133 \\
Drug intake ( $n)$ & 54 \\
Diagnosis & \\
Neurologic disorder $(n)$ & 40 \\
Back pain $(n)$ & 77 \\
Trauma $(n)$ & 44 \\
Tendinitis $(n)$ & 20 \\
Hip or knee replacement $(n)$ & 8 \\
Pain complaint $(n)$ & 115 \\
Chronic pain $\geq 6$ months $(n)$ & 101 \\
Orthopedic patients $(\mathrm{n})$ & 98 \\
Neurological patient $(\mathrm{n})$ & 17 \\
NRS (M + SD) & $5.6+2.4$ \\
Aim of treatment & \\
Pain relief $(n)$ & 47 \\
Functional recovery $(n)$ & 43 \\
Both $(n)$ & 98 \\
Catastrophic thinking & \\
None of the time $(n)$ & \\
Little/some of the time $(n)$ & \\
Most or all of the time & \\
\hline & \\
&
\end{tabular}

Parkinson's disease, extrapyramidal syndromes, multiple sclerosis, ataxic syndromes and peripheral neuropathy; the remaining $78.8 \%$ for orthopedic disorders: back pain $40.7 \%$, trauma $23.3 \%$, tendinitis $10.6 \%$, hip or knee replacement $4.2 \%$ (Fig. 1).

We were able to compare our case mix to that of all the 2008 patients treated in the same facility for the whole of the year 2010, finding no significant difference in terms of age (mean 66.5, SD 26.6, $p=0.140$ ), gender $(69.1 \%$ women, $p=0.716)$ and orthopedic vs neurological diagnosis $(79.2 \%$ vs $20.8 \%, p=0.699)$.

Table 1 also shows pain reports and patients' aims and expectations regarding treatment, as well as catastrophism. Of all patients, $60.9 \%$ complained of ongoing pain (mean NRS $=5.6 \pm 2.4$ ). In $95.8 \%$ of cases, the ongoing pain had been persistent for more than 3 months, in $87.8 \%$ for more than 6 months. Ongoing pain was reported by $66 \%$ of patients in orthopedic rehabilitation, but also by $40 \%$ of patients in neurological rehabilitation. According to $51 \%$ of patients answering to an open question, the main aims of undergoing treatment were both pain relief and functional recovery, while for $24.9 \%$ patients the main objective of undergoing treatment was pain relief, and for $22.8 \%$, functional recovery. For $50 \%$ of neurological patients, pain relief was the main goal of undergoing treatment, either included with functional recovery $(40 \%)$ or as the sole reason $(10 \%)$. Little or no confidence in treatment was reported by $15.3 \%$ of patients (11 neuromuscular, 10 spinal, 4 implants, 2 tendinitis, 1 trauma); catastrophic thinking was reported by $40.7 \%$ of patients; $28.6 \%$ of the patients were on pain medication during treatment ( 70.3 prescribed by a physician, 29.7 taken independently). Of those reporting chronic pain, only 10 persons had adhered to the psychological support group for patients with chronic pain available in the Department at the time of the interview (data not shown). Regarding the pain-related use of drugs (Fig. 2), $28.57 \%$ patients reported ongoing medication, divided into the following categories: paracetamol 7.41, $5.55 \%$ specific drugs for neuropathic pain, $25.93 \%$ opiates and $61.11 \%$ anti-inflammatory; $70 \%$ were taking drugs under medical prescription, while $30 \%$ were taking them on a self-prescribed basis (data not shown).

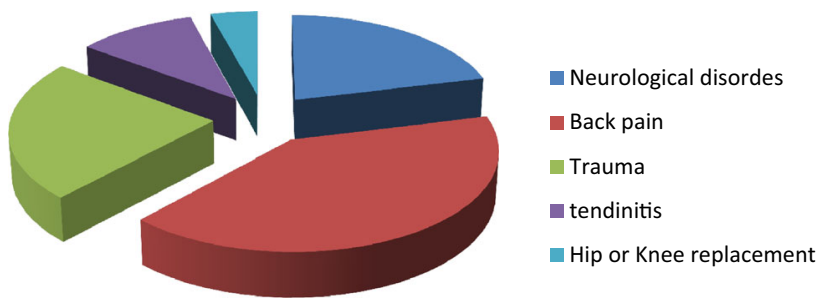

Fig. 1 Patients' diagnosis 


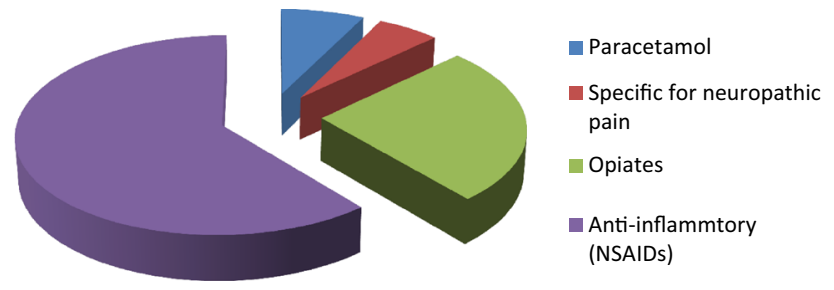

Fig. 2 Pain-related use of drugs

Table 2 Drug intake correlations

\begin{tabular}{ll}
\hline Drug intake correlations & $P$ \\
\hline Current pain & 0.007 \\
Higher pain intensity (NRS score) & 0.009 \\
Catastrophism & 0.009 \\
Advanced age & 0.0055 \\
Female gender & 0.724 \\
Reduced treatment expectations & 0.1 \\
\hline
\end{tabular}

Table 2 shows drug intake correlations. The use of drugs was related to advanced age $(p=0.005)$, to more intense pain $(p=0.009)$, and to the presence of catastrophism $p=0.0003)$, but not to treatment expectations $(p=0.10)$ or gender $(p=0.72)$.

Pain was more frequently reported in tendinitis (16/20) and back pain (62/87), less frequently in trauma (26/44), joint replacement (5/8), and neurological patients (16/40).

In a multivariate regression analysis, pain was independently correlated with orthopedic versus neurological diagnoses $(p=0.000)$, and to reduced treatment expectations $(p=0.020)$, while it was independent of age $(p=0.74)$ gender $(p=0.22)$, and catastrophism $(0.17)$ (Table 3).

\section{Discussion}

This study allowed the investigation of pain reports, the characteristics and correlations of the patients attending rehabilitation in the chosen outpatient department in the week of investigation; except for the few patients with severe cognitive impairment or aphasia, all persons attending rehabilitation were able and willing to answer the proposed self-administered questionnaire.

Our patients in outpatient rehabilitation reported a high occurrence of pain, reported in $61 \%$ of the cases, moderate in intensity on average, and, in the vast majority of cases, pain was lasting for more than 6 months (chronic pain). Indeed, by the less strict definition of chronic pain, lasting 3 months or more, almost all our patients reporting ongoing pain resulted in being affected by chronic pain [19].

Epidemiological data show that pain has an incidence of about $25-30 \%$ in the general European population. Italy is the country with the third highest prevalence of chronic pain (26\% of the whole Italian population) in Europe, after Norway and Belgium, and the country with the highest prevalence of severe chronic pain (13\%) [20]: one-fifth of the Italian population reports pain lasting for over 20 years, and one-third of these report pain as an ongoing basis that led them to leave work [21]. As expected, our results show that in our selected population of patients undergoing rehabilitation, reports of chronic pain are much higher than in the general population.

A rather unexpected finding was that their pain was almost invariably chronic. As chronic pain requires specific treatment, this finding, if confirmed in the multicentric study, would have relevant implications as to the appropriate means of pain management, including the possible implementation of a multidisciplinary rehabilitation team in outpatient rehabilitation facilities [22, 23]. Population studies show a higher prevalence of chronic pain in older persons [24] and in women, who complain of pain that is more severe, more frequent and longer lasting compared to men. This probably depends on some pain syndromes being age associated or gender associated. [25] In fact, in our population of patients attending rehabilitation, pain reports and severity were found to be independent of gender and age.

Despite pain being significantly more frequent among patients attending orthopedic rehabilitation (back pain, trauma, tendinitis, hip and knee implants), pain was also

Table 3 Independent correlations of current pain reports in the study population

\begin{tabular}{lccccc}
\hline & Odds Ratio & Std. Err & $\mathrm{z}$ & $p>|\mathrm{z}|$ & {$[95 \%$ Conf. Interval] } \\
\hline Age & 0.9966275 & 0.0103119 & -0.33 & 0.744 & $0.9766201-.017045$ \\
Gender & 0.6426114 & 0.2331941 & -1.22 & 0.223 & $0.3155441-1.30869$ \\
Diagnosis neurological & 0.2262941 & 0.093295 & -3.60 & $\mathbf{0 . 0 0 0}$ & $0.1008661-0.5076927$ \\
Catastrophic thinking & 158.339 & 0.5308322 & 1.37 & 0.170 & $0.8207716-3.054595$ \\
Expectations & 0.2912362 & 0.1541109 & -2.33 & $\mathbf{0 . 0 2 0}$ & $0.1032339-0.8216146$
\end{tabular}

N. obs $=189$ LR chi2 $(5)=19.44$ Prob.chi2 $=0.0016$ pseudoR2 $=0.0783$ Log likelihood $=-114.42279$

Bold values are statistically significant at $p<0.05$ 
reported by $42.5 \%$ of neurological patients. Indeed, chronic pain in neurological patients is a common symptom, but still very much neglected [26]. Our results are consistent with studies reporting as many as $39.8 \%$ of patients with multiple sclerosis having major pain that can have serious repercussions on their quality of life [27]. A study conducted in Norway reports that $83 \%$ of patients with Parkinson's disease suffer pain, and that they had an increased level of pain compared to the general population [28], while a Danish study reports chronic pain in $55 \%$ of patients who survive a stroke [29]. These results, if confirmed in the multicentric study, would imply a recommendation that more attention be devoted to pain treatment and management in neurological rehabilitation. A declared objective of our investigation was to focus on patients' perspectives and expectations: the relief from pain, alone or in combination with functional recovery, was considered to be the main therapeutic goal of the rehabilitation treatment by most patients reporting pain, including also half of the patients with a neurological disorder. This result stimulates a reflection on the importance of pain assessment and monitoring during the whole rehabilitation process [30]. Indeed, a study of Elizabeth et al. concerning patient attitudes regarding pain in a rehabilitation hospital shows that pain is judged by about half of the patients to be a significant negative factor impeding rehabilitation efforts [31]. However, despite the introduction of the biopsychosocial model of the ICF (International Classification of Functioning, Disability and Health), [5] the dominant therapeutic approach is still very often problem-centered rather than patient-centered. In Italy, Law No. 38 dated 15 March 2010 upholds the right not to suffer, and for all citizens to have access to pain relief. This law represents a real revolution in the understanding and management of pain in that it imposes an obligation for detecting and monitoring the patient's pain when being admitted to a health facility [32]. Concerning pain management, a treatment goal for some rehabilitation programs is a reduction in the use of pain-related medication [33]. In our sample, $28.57 \%$ of patients took drugs divided into the following categories: paracetamol 7.41, 5.55\% specific drugs for neuropathic pain, $25.93 \%$ opiates and $61.11 \%$ anti-inflammatory. Such a distribution shows a seemingly disproportionate use of anti-inflammatory drugs or opiates, rather than paracetamol, which is indicated as the firstchoice drug for treatment of chronic pain [34], but our sample is indeed rather heterogeneous in terms of diagnosis, and such numbers do not allow a separate analysis. Use of drugs was related to more intense pain, to catastrophism, and to advanced age. As both non-steroid antiinflammatory drugs and opiates have been reported to show more frequent and more serious side effects in the elderly [35], the relationship with pain medication intake and increasing age is a relevant issue in rehabilitation. Another issue is the source of prescription-type drugs for pain relief. Patients may consume self-prescribed medications because of unrealistic expectations for pain relief, increased reliance on medications, and inadequate pain education [36]. Indeed, habituation and failure of previously effective medications may even lead to drug overuse [37]. A recent American study suggests that up to 55.9 of those using painkillers reported having received the drug from a relative or friend rather than receiving a specific medical prescription [38]. Our data seem to suggest a lower ratio of non-medically prescribed drug intake, but still, even if these figures are confirmed in the multicentric study, the risk of drug abuse and of uncontrolled exposure to drug side effects is a serious issue that also should be taken into account in a rehabilitation setting.

As we were focused on performing a rapid screening of pain occurrence and characteristics at this stage of the project, we did not perform a thorough assessment of disability nor of the psychosocial factors known to be associated with chronic pain, such as anxiety, depression, and low socioeconomic status, and this is definitely a limitation of our study [8]. Further, we did not investigate the possible correlation of pain with the functional outcome of the rehabilitation intervention. However, we did find that $40 \%$ of our patients report catastrophic thinking. Thus, introducing a biopsychosocial perspective to pain, and operating within a framework of multidisciplinary pain rehabilitation, may improve the effectiveness of rehabilitation programs in patients experiencing chronic pain with pain relief as their primary objective [30]. Nevertheless, only 10 patients were attending the psychological support group that was offered to all patients with chronic pain attending the facility, thus suggesting that this kind of treatment is not always easily accepted by patients seeking physical rehabilitation.

This study presents some limitations. First and foremost, our results are limited to the case mix of patients attending the outpatient department in a given time frame, so they cannot be considered representative of the general patient population undergoing outpatient rehabilitation. Even so, this case mix was representative of the age, gender and diagnosis of the general population attending the department in the same year. More generalizable results should be obtained by a multicentric study involving inpatients and more facilities.

Our purpose was to investigate pain by posing a short set of questions that could be self-administered and widely used in clinical practice. Indeed, excluding only patients with dementia and aphasia who require a specific pain assessment [39], we were able to enroll 189 out of 210 outpatients, none of whom refused to answer the self-administered questionnaire. This led us to select only the first 
three questions from the BPI, instead of adopting the whole questionnaire or using another multimodal tool. In accordance with the BPI, we measured pain severity by a numeric rating scale, that has been shown to have the same psychometric properties as the Visual Analog Scale, and that we found easier to record and measure [40]. For the same reason, we did not include in our assessment a screening tool for neuropathics vs nociceptive pain, which could be introduced as a second-level assessment.

Finally, as already mentioned, we did not thoroughly investigate the occurrence of anxiety and depression and life satisfaction that may have provided us more insight into a correlation with pain [41]. Moreover, while exploring catastrophism, which has been shown to be strongly associated with pain as well as with a poor rehabilitation outcome [42], we used only a single question rather than the complete tool [18], thus possibly limiting the sensitivity and specificity of our findings in this respect.

\section{Conclusion}

A very high prevalence of chronic pain was observed in this sample of patients undergoing outpatient rehabilitation. Pain relief, with or without functional recovery, is considered the main goal of treatment by $75 \%$ of all patients in pain (50\% of neurological patients). The use of pain medication is related to pain intensity, old age and catastrophism, while the pain complaint is independently correlated with an orthopedic vs neurological diagnosis, and to reduced treatment expectations, but not to age or gender.

Acknowledgements Many thanks to all patients and all the researchers at the Don Gnocchi center in Florence who participated in the study. Role of the funding source Ministry of Health.

Conflict of interest The manuscript submitted does not contain information about medical device(s)/drug(s). Institutional funds were received in support of this work. No benefits in any form have been or will be received from a commercial party related directly or indirectly to the subject of this manuscript. The authors declare no conflict of interest. This work has not been previously presented in any form.

\section{References}

1. Maxey L, Magnusson J (2001) Rehabilitation for the postsurgical orthopedic patient. Mosby inc, Missouri USA

2. Mersky H, Bogduk N (1994) Classification of pain. International Association for the Study of Pain: IASP pain terminology. Seattle, IASP Task Force on Taxonomy, 2nd edn, pp 209-214

3. Bloodworth D, Calvillo C, Smith K, Grabois M (2000) Chronic pain syndromes: evaluation and treatment. In: Braddom RL: Physical Medicine Rehabilitation 2nd edn. Philadelphia: WB Saunders

4. Jansen MJ, Hendriks EJ, Oostendorp RA, Dekker J, De Bie RA (2010) Quality indicators indicate good adherence to the clinical practice guideline on "Osteoarthritis of the hip and knee" and few prognostic factors influence outcome indicators: a prospective cohort study. Eur J Phys Rehabil Med 46:337-345

5. ICF Classificazione Internazionale del Funzionamento (2002) della Disabilità e della salute. OMS. Edizioni Erickson, Gardolo TN

6. Merry B, Campbell CM, Buenaver LF, McGuire L, Haythornthwaite JA, Doleys DM, Edwards RR (2011) Ethnic group differences in the outcomes of multidisciplinary pain treatment. J Musculoskeletal Pain 19(1):24-30

7. Gordon DB, Dahl JL (2004) Quality improvement challenges in pain management. Pain 107:1-4

8. Langley P, Müller-Schwefe G, Nicolaou A, Liedgens H, Pergolizzi J, Varrassi G (2010) The societal impact of pain in the European Union: health-related quality of life and healthcare resource utilization. J Med Econ 13(3):571-581

9. Loeser JD, Treede RD (2008) The Kyoto protocol of IASP basic pain terminology. Pain 137(3):473-477

10. Von Korff M, Wagner EH, Dworkin SF, Saunders KW (1991) Chronic pain and use of ambulatory health care. Psychosom Med 53:61-79

11. Merrick D, Siolund BH (2009) Patients' pretreatment beliefs about recovery influence outcome of a pain rehabilitation program. Eur J Phys Rehabil Med 45:391-401

12. Folstein MF, Folstein SE, McHugh PR (1975) 1975; Mini Mental State: a practical method for grading the cognitive state of patient for the clinician. J Psych Res 12:189-198

13. Caraceni A, Mendoza TR, Mercaglia E, Baratella C, Edwars K, Serlin CS (1999) A validation study of an Italian version of the rief pain inventory. Pain 65:87-92

14. Childs J, Piva S, Fritz J (2005) Responsiveness of the Numeric pain Rating Scale in patients with low back pain. Spine 30:1331-1334

15. Downie WW, Leatham PA, Rhind VM, Wright V, Branco JA, Anderson JA (1978) Studies with pain rating scales. Ann Rheum Dis $37: 378-381$

16. Cecchi F, Debolini P, Molino Lova R, Macchi C, Bandinelli S, Bartali B, Lauretani F, Benvenuti E, Hicks G, Ferrucci L (2006) Epidemiology of back pain in a representative cohort of Italian persons 65 years of age and older: the InCHIANTI study. Spine 31:1149-1155

17. Sullivan MJL, Bishop SR, Pivik J (1995) The pain catastrophizing scale: development and validation. Psychol Assess 7:524-532

18. Cecchi F, Molino-Lova R, Paperini A, Boni R, Castagnoli C, Gentile J, Pasquini G, Macchi C (2011) Predictors of short- and long-term outcome in patients with chronic non-specific neck pain undergoing an exercise-based rehabilitation program: a prospective cohort study with 1-year follow-up. Intern Emerg Med 6(5):413-421

19. Weiner DK (2007) Office management of chronic pain in the elderly. Am J Med 120:306-315

20. Breivik H, Cherny N, Collett B, de Conno F, Filbet M, Foubert AJ, Cohen R, Dow L (2009) Cancer-related pain: a pan-European survey of prevalence, treatment, and patient attitudes. Ann Oncol 20(8):1420-1433

21. Associazione Italiana per lo Studio del Dolore (Aisd), GfK Eurisko, 2007

22. Airaksinen O, Brox JI, Cedraschi C (2006) European guidelines for the management of chronic nonspecific low back pain. Eur Spine J 15:S192-S300

23. Koes BW, van Tulder MW, Thomas S (2010) Diagnosis and treatment of low back pain. BMJ Clin Rev 332:1430-1434

24. Raftery M, De la Harpe D, Murphy AW, Normand C, Sarma K, McGuire B (2011) Chronic pain in the Republic of Irelandcommunity prevalence, psychosocial profile and predictors of 
pain-related disability: results from the PRIME study, Part 1. Pain 152:1096-1103

25. Fillingim RB (2000) Sex, gender, and pain: women and men really are different. Curr Rev Pain 4:24-30

26. Boneschi FM, Colombo B, Annovazzi P (2008) Lifetime and actual prevalence of pain and headache in multiple sclerosis. Mult Scler 14(4):514-521

27. Barnes D (2000) Multiple Sclerosis. Questions and Answers. Merit Publishing International

28. Beiske AG, Loge JH, Ronningen A, Svensson E (2009) Pain in Parkinson's disease: prevalence and characteristic's? Pain 141:173-177

29. Klit H, Brix Finnerup, Andersen G, Jensen TS (2011) Central poststroke pain: a population-based study. Pain 152:818-824

30. Moseley L (2002) Combined physiotherapy and education is efficacious for chronic low back pain. Austr J Physiother 48(4):297-302

31. Elizabeth W, Loder, Alan B (2002) A Pilot study of pain and attitudes toward pain in a rehabilitation population. Pain Med 3(4):333-338

32. Gazzetta Ufficiale-Serie Generale n. 65, March 19, 2010

33. Cunningham JL, Rome JD, Kerkvliet JL, Townsend CO (2009) Reduction in medication costs for patients with chronic nonmalignant pain completing a pain rehabilitation program: a prospective analysis of admission, discharge, and 6-month follow-up medication costs. Pain Med 10(5):787-796

34. WHO (2009) Cancer pain relief. 2nd edn. 1996; American Geriatrics Society Panel. Management of Persistent Pain in Older Persons. JAGS 57:1331-1346

35. Salomon DH, Rassen JA, Glynn RJ, Garneau K, Levin R, Lee J, Schneeweiss S (2010) The comparative safety of opioids for nonmalignant pain in older adults. Arch Int Med 170(22):1979-1986
36. Taylor ML (2011) The impact of the "business" of pain medicine on patient care. Pain Med 12(5):763-772

37. Geppetti Pierangelo, De Cesaris Francesco, Nicoletti Paola, Benemei Silvia (2010) Chronic headaches and medication overuse. IEM 5(1):7-11

38. Manchikanti L, Boswell MV, Singh V, Benyamin RM, Fellows B, Abdi S, Buenaventura RM, Conn A, Datta S, Derby R, Falco FJE, Erhart S, Diwan S, Hayek SM, Helm S, Parr AT, Schultz DM, Smith HS, Wolfer LR, Hirsch JA (2009) Comprehensive evidence-based guidelines for interventional techniques in the management of chronic spinal pain. Pain Phys 12:699-802

39. Costardi D, Rozzini L, Costanzi C, Ghianda D, Franzoni S, Padovani A, Trabucchi M (2007) The Italian version of the pain assessment in advanced dementia (PAINAD) scale. Arch Gerontol Geriatr 44(2):175-180

40. Hjermstad MJ, Fayers PM, Haugen DF, Caraceni A, Hanks GW, Loge JH, Fainsinger R, Aass N, Kaasa S (2011) European Palliative Care Research Collaborative (EPCRC). studies comparing numerical rating scales, verbal rating scales, and visual analogue scales for assessment of pain intensity in adults: a systematic literature review. J Pain Symp Manage 41(6):1073-1093

41. Pulvers K, Hood A (2013) The role of positive traits and pain catastrophizing in pain perception. Curr Pain Headache Rep 17(5):330

42. Radat F, Koleck M (2011) Pain and depression: cognitive and behavioural mediators of a frequent association. Encephale. 37(3):172-179 